Pacific Journal of Mathematic 


\title{
A COMPARISON THEOREM FOR EIGENVALUES OF NORMAL MATRICES
}

\author{
KY FAN
}

The following interesting theorem was recently obtained by $\mathrm{H}$. Wielandt (Oral communication, see also J. Todd [3]):

Let $M, N$ be two normal matrices of order $n$, and let $r$ denote the rank of $M-N$. Let $D$ be an arbitrary closed circular disk in the complex plane, If $D$ contains exactly $p$ eigenvalues of $M$, and exactly $q$ eigenvalues of $N$, then $|p-q| \leq r$.

It is then natural to raise the following question: Without considering the rank of $M-N$, is it possible to compare the eigenvalues of $M$ and $N$ in a manner similar to that of Wielandt's theorem? The purpose of this Note is to present such a rank-free comparison theorem which includes Wielandt's theorem stated above.

Theorem. Let $M, N$ be two normal matrices ${ }^{1}$ of order $n$ and let $r$ be an integer such that $0 \leq r<n$. Let $\varepsilon \geq 0$ be such that $\varepsilon^{2}$ is not less than the $(r+1)$ th eigenvalue of $(M-N)^{*}(M-N)$, when the eigenvalues of $(M-N)^{*}(M-N)$ are arranged in descending order. ${ }^{2}$ If a closed circular disk

$$
\left|z-z_{0}\right| \leq \rho
$$

contains $p$ eigenvalues of $M$, then the concentric disk

$$
\left|z-z_{0}\right| \leq \rho+\varepsilon
$$

contains at least $p-r$ eigenvalues of $N$.

While Wielandt's proof of his theorem uses geometric arguments involving convexity, the proof of our theorem will be based on an inequality (Lemma below). This difference in methods explains why our result is of more quantitative character than Wielandt's theorem.

Lemma. Let $A, B$ be any two matrices ${ }^{3}$ of order n. If $\left\{\alpha_{i}\right\},\left\{\beta_{i}\right\}$, $\left\{\gamma_{i}\right\}$ are the eigenvalues of $A^{*} A, B^{*} B$ and $(A+B)^{*}(A+B)$ respectively, each arranged in descending order

Received August 1, 1954. This paper was prepared in part under a National Bureau of Standards contract with The American University, sponsored by the Office of Scientific Research of the Air Research and Development Command, USAF.

1 The elements of all matrices considered here are real or complex numbers.

${ }^{2}$ As usual, the adjoint of a matrix $A$ is denoted by $A^{*}$.

3 Here $A, B$ need not be normal. 


$$
\alpha_{i} \geq \alpha_{i+1}, \quad \beta_{i} \geq \beta_{i+1}, \quad \gamma_{i} \geq \gamma_{i+1},
$$

then the inequality

$$
\sqrt{\gamma_{i+j+1}} \leq \sqrt{\alpha_{i+1}}+\sqrt{\beta_{j+1}}
$$

holds for any two nonnegative integers $i, j$ such that $i+j+1 \leq n$.

A more general form of this lemma (valid for completely continuous linear operators in a Hilbert space) has been given in [2], and is a generalization of a classical inequality of $\mathrm{H}$. Weyl [4, p. 445] concerning eigenvalues of sum of two symmetric kernels of linear integral equations.

Proof of the theorem. Let $\left\{\mu_{i}\right\},\left\{\nu_{i}\right\}$ denote the eigenvalues of $M$, $N$ respectively and so arranged that

$$
\left|\mu_{i}-z_{0}\right| \geq\left|\mu_{i+1}-z_{0}\right|, \quad\left|\nu_{i}-z_{0}\right| \geq\left|\nu_{i+1}-z_{0}\right|, \quad(1 \leq i \leq n-1) .
$$

Let

$$
A=M-z_{0} I, \quad B=N-M .
$$

Then $A+B=N-z_{0} I$. Let $\left\{\alpha_{i}\right\},\left\{\beta_{i}\right\},\left\{\gamma_{i}\right\}$ denote the eigenvalues of $A^{*} A, B^{*} B$ and $(A+B)^{*}(A+B)$, each arranged in descending order. As $M, N$ are both normal, we have

$$
\alpha_{i}=\left|\mu_{i}-z_{0}\right|^{2}, \quad \gamma_{i}=\left|\nu_{i}-z_{0}\right|^{2},
$$

By the above Lemma, we have

$$
\left|\nu_{i+r}-z_{0}\right| \leq\left|\mu_{i}-z_{0}\right|+\sqrt{\beta_{r+1}},
$$

Using our hypothesis $\beta_{r+1} \leq \varepsilon^{2}$, we obtain

$$
\left|\nu_{i+r}-z_{0}\right| \leq\left|\mu_{i}-z_{0}\right|+\varepsilon,
$$

Let $p$ denote the number of eigenvalues $\mu_{i}$ of $M$ contained in the disk $\left|z-z_{0}\right| \leq \rho$, and $q$ the number of eigenvalues $\nu_{i}$ of $N$ contained in the concentric disk $\left|z-z_{0}\right| \leq \rho+\varepsilon$. We shall prove that

$$
q \geq p-r .
$$

If $n-q-r<1$, then $q \geq n-r \geq p-r$. Thus we may assume $1 \leq n-$ $q-r$. By (1),

$$
\left|\nu_{n-q}-z_{0}\right| \leq\left|\mu_{n-q-r}-z_{0}\right|+\varepsilon .
$$

But, according to the definition of $q$, we have

$$
\left|\nu_{n-q}-z_{0}\right|>\rho+\varepsilon \text {. }
$$


Therefore

$$
\left|\mu_{i i-\eta \ldots \rho}-z_{0}\right|>\rho,
$$

which implies $n-q-r \leq n-p$ or (2). Our theorem is thus proved.

Corollary. Let $M, N$ be two normal matrices of order $n$ and let $r$ be an integer such that $0 \leq r<n$. Let $x_{1}, x_{2}, \cdots, x_{n-r}$ be $n-r$ orthonormal vectors in the unitary $n$-space. If a closed circular disk $\left|z-z_{0}\right| \leq \rho$ contains $p$ eigenvalues of $M$, then the concentric disk

$$
\left|z-z_{0}\right| \leq \rho+\left(\sum_{i=1}^{n-r}\left\|(M-N) x_{i}\right\|^{2}\right)^{\frac{1}{2}}
$$

contains at least $p-r$ eigenvalues of $N$.

Proof. By a minimum property of eigenvalues of Hermitian matrices [1, Theorem 1], the expression

$$
\sum_{i=1}^{n-r}\left\|(M-N) x_{i}\right\|^{2}=\sum_{i=1}^{n-r}\left((M-N)^{*}(M-N) x_{i}, x_{i}\right)
$$

is not less than the sum of the last $n-r$ eigenvalues of $(M-N)^{*}(M-N)$, and consequently not less than the $(r+1)$ th eigenvalue of $(M-N)^{*}(M$ $-N)$. Thus the corollary follows directly from the theorem.

In case $r$ is the rank of $M-N$, we can choose $n-r$ orthonormal vectors $x_{1}, x_{2}, \cdots, x_{n-r}$ such that

$$
(M-N) x_{i}=0
$$$$
(1 \leq i \leq n-r) .
$$

Then the disk (3) becomes $\left|z-z_{0}\right| \leq \rho$ and the corollary reduces to Wielandt's theorem.

\section{REFERENCES}

1. K. Fan, On a theorem of Weyl concorning eigenvalues of linear transformations, $I$, Proc. Nat. Acad. Sci., 35 (1949), 652-655.

2. Maximum properties and inequalities for the eigenvalues of completely continuous operators, Proc. Nat. Acad. Sci., 37 (1951), 760-766.

3. J. Todd, The condition of certain matrices II, Archiv der Math., 5 (1954), 249-257.

4. H. Weyl, Das asymptotische Verteilungsgesetz der Eigenwerte linearer particller Differentialgleichungen, Math. Ann., 71 (1912), 441-479.

The American University AND

UNIVERSITY OF NOTRE DAME 

.././. ./FrontMatter/paper .pdf 


\section{Pacific Journal of Mathematics}

Nesmith Cornett Ankeny and Theodore Joseph Rivlin, On a theorem of S.

Bernstei........................................ 849

Louis Auslander, The use of forms in variational calculation .......... 853

Paul Civin, Abstract Riemann sum . .......................... 861

Paul Civin, Some ergodic theorems involving two operator ............ 869

Eckford Cohen, The number of solutions of certain cubic congruence .... . 877

Richard M. Cohn, Specializations over difference field .............. 887

Jean Dieudonné, Pseudo-discriminant and Dickson invarian . . ......... 907

Ky Fan, A comparison theorem for eigenvalues of normal matrice ........ 911

Richard P. Gosselin, On the convergence behaviour of trigonometric interpolating polynomial ........................... 915

Peter K. Henrici, On generating functions of the Jacobi polynomial . . . . . . . 923

Meyer Jerison, An algebra associated with a compact grou ............ 933

Wilhelm Magnus, Infinite determinants associated with Hill's equatio . . . . . 941

G. Power and D. L. Scott-Hutton, The slow steady motion of liquid past a semi-elliptical bos................................. 953

Lyle E. Pursell, An algebraic characterization of fixed ideals in certain function ring .................................... 963

C. T. Rajagopal, Additional note on some Tauberian theorems of O. Szás . . 971 Louis Baker Rall, Error bounds for iterative solutions of Fredholm integral

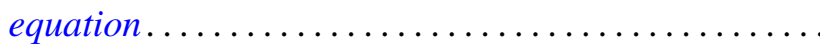

Shigeo Sasaki and Kentaro Yano, Pseudo-analytic vectors on

pseudo-Kählerian manifold ......................

Eugene Schenkman, On the tower theorem for finite group

P. Stein and John E. L. Peck, On the numerical solution of Poisson's equation over a rectangl ........................

Morgan Ward, The mappings of the positive integers into themselves which preserve divisio .

Seth Warner, Weak locally multiplicatively-convex algebra 1025

Louis Weisner, Group-theoretic origin of certain generating function .... 